\title{
¿Qué información tienen los pacientes ancianos con insuficiencia cardiaca sobre su tratamiento?
}

\author{
P. GARCÍA GÓMEZ, C. MONTOTO OTERO, N. REYES PERERA, \\ I. GARCÍA SÁNCHEZ, P. AGUDO
}

Servicio de Medicina Interna I. Hospital General Universitario Gregorio Marañón. Madrid

WHAT INFORMATION HAVE OLD PATIENTS WITH HEART FAI LURE ABOUT THEIR TREATMENT?

\section{RESUMEN}

Introducción: La insuficiencia cardiaca (IC) es uno de los principales motivos de ingreso hospitalario, viéndose favorecido este hecho por la falta de adherencia al tratamiento de los pacientes. Este estudio fue diseñado para conocer el grado de información sobre la IC con el que cuentan los pacientes.

Pacientes y métodos: Se realizó entrevista personalizada a 75 pacientes ingresados con diagnóstico de IC sobre sus conocimientos sobre la enfermedad y el tratamiento que seguían.

Resultados: Losc pacientes consideraron motivo de consulta el aumento de peso, la disnea y los edemas (99\%), entendiendo como necesario el control del peso para su estabilización (82\%). Hasta un 50\% no considera importante el control de la diuresis ni el de ingesta hídrica. Ven en el ejercicio físico moderado y la restricción de sal un beneficio $(85 \%$ y $100 \%$ respectivamente). El $24 \%$ de los pacientes consideró que podían utilizar cualquier tipo de antiinflamatorio no esteroideo (AINEs). Un $79 \%$ de los pacientes reconocen el efecto diurético de la furosemida y un $70 \%$ atribuyen a la digoxina su efecto cardiológico, mientras que tan sólo conocen la utilidad de los Inhibidores de la Enzima Convertidora de Angiotensina (IECAs) un 46\%. Los efectos secundarios de dichos fármacos son, en general, desconocidos $(56 \%, 65 \%$ y $80 \%$ respectivamente).

Conclusiones: El paciente con IC posee escasa información sobre el tratamiento y medidas higiénico-dietéticas relacionadas con su enfermedad, lo que confirma la dificultad de estos pacientes para el cuidado adecuado de su patología. Se debería incidir en este punto para evitar el reingreso de estos pacientes por IC.

PALABRAS CLAVE: Insuficiencia cardiaca. Información. Tratamiento.

\begin{abstract}
Introduction: Heart failure is one of the leading cause of hospital admision because of the lack of adherence to treatment of these patients. This study was designed to know patients knowledge about heart failure. Patients and methods: 75 patiens admited in ward with the diagnosis of heart failure were asked about the illness and the treatment they took.

Results: Patiens considered weight gain, dyspnea and edemas a cau se of consultant to their doctors (99\%). They understood as necesary the weight control to their stabilization ( $82 \%$ ).

One half of the interviewed did not consider important the control of diuresis and water ingestion. Most of the patients thought that mild phy sical exercise and sodium restriction could improve their physical status ( $85 \%$ and $100 \%$ respectively). $24 \%$ of the patiens considered they could use any kind of non steroid anti-inflammatory drug. $79 \%$ of the patients knew the diuretic effect of furosemida and $70 \%$ the cardiologic effect of digoxina, all thought only just $46 \%$ recognized the use of angiotensin converse enzyme inhibitors for heart failure and blood presure. The adverse events of these drugs were unknow $(56 \%, 65 \%$ and $80 \%$ respectively).

Conclusions: Patients with heart failure had poor information about dietary and medical treatment. This could expain the problems their have for the optimal use of effective medication, being poor patients adheren ce to pharmacologic and dietary therapy a common cause of exacerba tion.

It would be necesary to increase patients information to avoid heart failure progression and descompensation.
\end{abstract}

KEY WORDS: Heart failure. Information. Treatment.

García Gómez P, Montoto Otero C, Reyes Perera N, García Sánchez I, Agudo P. ¿Qué información tienen los pacientes ancianos con insuficiencia cardiaca sobre su tratamiento?. An Med Interna (Madrid) 2003; 20: 59-62.

\section{INTRODUCCIÓN}

En las últimas décadas se ha modificado el conocimiento y la comprensión de la fisiopatología y epidemiología de la insuficiencia cardiaca $(1,2)$ así como de los factores que la favorecen, lo que ha introducido grandes cambios en los tratamientos farmacológicos y su manejo general.

Aunque la IC es frecuente, los estudios epidemiológicos en España son escasos, ya que la epidemiología ha profundizado más en las causas etiológicas que en la propia IC. Apro-

Trabajo aceptado: 19 de octubre de 2002

Correspondencia: Carmen Montoto Otero. Servicio Medicina Interna I. Hospital Gregorio Marañón. C/ Doctor Esquerdo Nº 46. 28007 Madrid. 
ximadamente se estima que el $1 \%$ de la población puede presentar IC (estudio Framingham) (3). El 1 a $2 \%$ de los ingresos en un hospital general son por IC (4), de tal manera que podría asumirse que más de 500.000 ingresos hospitalarios son motivados por dicha enfermedad. Hasta el momento actual la hospitalización de estos pacientes se relacionaba fundamentalmente con la existencia de una serie de factores de riesgo bien definidos, dejando en el olvido el desconocimiento que el propio paciente tiene sobre las medidas higiénico-dietéticas y el manejo terapéutico de su enfermedad, así como de otros factores psicosociales que en muchas ocasiones les obligan a reingresar $(5,6)$.

La finalidad de este estudio, por todo ello, es conocer el grado de información que poseen los pacientes diagnosticados de IC acerca de su enfermedad, las medidas higiénico-dietéticas y el tratamiento de la misma, para poder adecuar la planificación del tratamiento y seguimiento de estos pacientes.

\section{MATERIAL Y MÉTODOS}

Se realizó una entrevista personalizada a 75 pacientes consecutivos mayores de 65 años incluidos entre todos los ingresados en el Hospital General Universitario Gregorio Marañón con diagnóstico de insuficiencia cardiaca en el servicio de Medicina Interna. Los pacientes eran descartados si tenían factores que impidieran la adecuada comprensión de las preguntas y no tuvieran además algún familiar presente de los ocupados de su cuidado. El diagnóstico de insuficiencia cardiaca fue establecido por la presencia de datos clínicos sugerentes de la misma, según los criterios de Framingham (3) y habiéndose realizado a cada paciente un ecocardiograma. Se expusieron las preguntas de forma sencilla (Tabla I), comprobando, en cada una de ellas, la correcta comprensión por parte del paciente o su familiar. La entrevista se centró en determinar el grado de conocimiento del paciente sobre las medidas higiénico-dietéticas y el manejo farmacológico de su enfermedad.

Se realizó análisis de tipo descriptivo, interpretando los resultados obtenidos del mismo a través del cómputo de las respuestas emitidas por los pacientes o sus familiares, recogiendo estadísticamente la frecuencia de afirmaciones hacia las distintas preguntas.

\section{RESULTADOS}

De las 75 entrevistas realizadas, 42 pacientes fueron mujeres y 33 varones, la edad media fue 76,81 $\pm 10,5$ años. En referencia a las preguntas relacionadas con los aspectos higiénico-dietéticos de la enfermedad se obtuvieron los siguientes resultados: el $82 \%$ de la población estudiada consideró como algo necesario para la estabilización de su enfermedad de base el hecho de controlar su peso, mientras que tan sólo un $12 \%$ de los mismos pensaban que esta medida no era necesaria y un $5 \%$ desconocían la utilidad de la misma. El 85\% consideró al ejercicio moderado y el $100 \%$ el uso restringido de sal como algo beneficioso. Por el contrario, un $50 \%$ de los pacientes encuestados no identificaron el control de la diuresis y la ingesta hídrica moderada como medidas preventivas para el desarrollo de la insuficiencia cardiaca, y tan sólo reconocieron estas medidas como efectivas un $37 \%$ y un $24 \%$ de los
TABLA I

CUESTIONARIO REALIZADO A LOS

PACIENTES ENCUESTADOS

¿Se debe consultar al médico ante la presencia de un aumento de peso, fatiga $\mathrm{y} / \mathrm{o}$ edemas?

¿Se debe controlar el peso para que no aumente ni disminuya?

¿Es bueno para el corazón la realización de ejercicio físico moderado?

¿Debo restringir el uso de sal en las comidas?

¿Es importante vigilar la diuresis?

¿Debo moderar la ingesta hídrica?

¿Cuál es el uso de la furosemida?

¿Sabe que la digoxina es un medicamento para el corazón?

Sobre los IECAS, ¿Conoce su uso para la tensión arterial y el corazón?

¿Sabe que la furosemida le puede producir calambres como efecto secundario?

¿Reconoce las náuseas y la inapetencia como efectos secundarios de la digoxina?

¿Son el mareo y la tos efectos secundarios de los IECAS?

encuestados respectivamente, desconociendo esta información un $16 \%$ y un $24 \%$. En relación a los aspectos clínicos sobre los que la población fue interrogada el $99 \%$ consultaría al médico ante la presencia de disnea y/o edemas de miembros inferiores (Tabla II).

En cuanto a los conocimientos del paciente acerca del manejo farmacológico de la insuficiencia cardiaca el $24 \%$ de los entrevistados opinó que podía utilizar cualquier tipo de Antiinflamatorio No Esteroideo (AINE) para el manejo de dolor, independientemente de su enfermedad. El 28\% reseñó que no conocía si podía utilizarlos o no y un $48 \%$ reconoció que no debía utilizar este grupo de fármacos sin consultar con su médico de cabecera. Un $84 \%$ de los pacientes en tratamiento con furosemida la identificaron como diurético y un $15 \%$ desconocían su efecto. El $70 \%$ de los pacientes en tratamiento con digoxina la identificaron como medicamento útil para el manejo de su enfermedad y un $30 \%$ desconocían su efecto. Un 54\% de los encuestados no reconocía a los IECAS como tratamiento de su enfermedad (Fig. 1). En el campo de las reacciones adversas secundarias a la utilización de dichos fármacos, de los que utilizaban furosemida un $22 \%$ negaron y el $56 \%$ no reconocieron la posible aparición de calambres en relación con el uso de la misma, un $65 \%$ de los pacientes en tratamiento con digoxina no identificaron las náuseas y la inapetencia como posibles efectos secundarios a su utilización y un $22 \%$ lo negaron. Por último, un $80 \%$ de los que tomaban IECAS desconocían la posible aparición de mareo y tos como complicación de su ingesta (Fig. 2). 
TABLA II

M EDIDAS HIGIÉNICO -DIETÉTICAS EN INSUFICIENCIA CARDIACA

\begin{tabular}{lccccccc}
\hline $\begin{array}{l}\text { Consultaría si } \\
\text { edemas o fatiga? }\end{array}$ & $\begin{array}{c}\text { ¿Es bueno el } \\
\text { control del peso? }\end{array}$ & $\begin{array}{c}\text { ¿Es bueno } \\
\text { el ejercicio? }\end{array}$ & $\begin{array}{c}\text { ¿Debemos restringir } \\
\text { el consumo de sal? }\end{array}$ & $\begin{array}{c}\text { ¿Hay que moderar } \\
\text { el consumo de agua? }\end{array}$ & $\begin{array}{c}\text { ¿Debemos vigilar } \\
\text { la diuresis? }\end{array}$ & $\begin{array}{c}\text { ¿Puedo tomar } \\
\text { cualquier AlNE? }\end{array}$ & $\%$ \\
\hline 99 & 82 & 85 & 100 & 24 & 27 & 24 & SI \\
1 & 12 & 14 & 0 & 52 & 46 & 48 & NO \\
0 & 5 & 1 & 0 & 24 & 16 & 28 & NS/NC \\
\hline
\end{tabular}

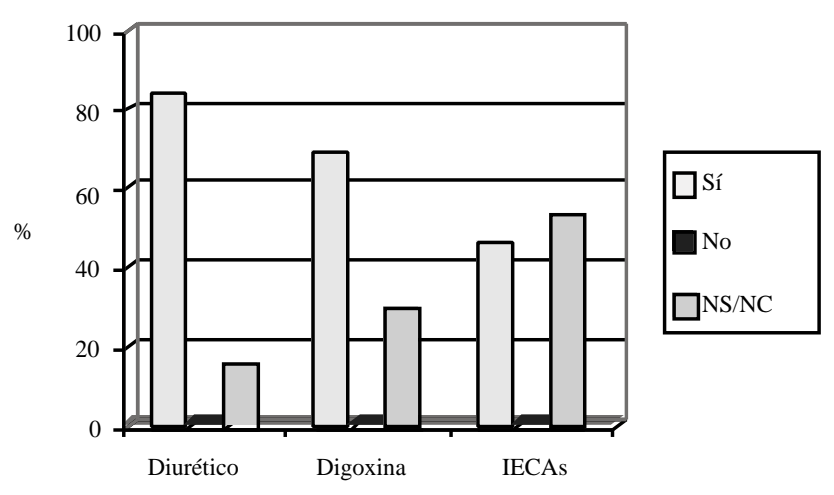

Fig. 1. Conocimiento sobre efecto de distintos fármacos en el tratamiento de la IC (" ¿sabe cuál es el efecto del diurético, digoxina e IECAs?").

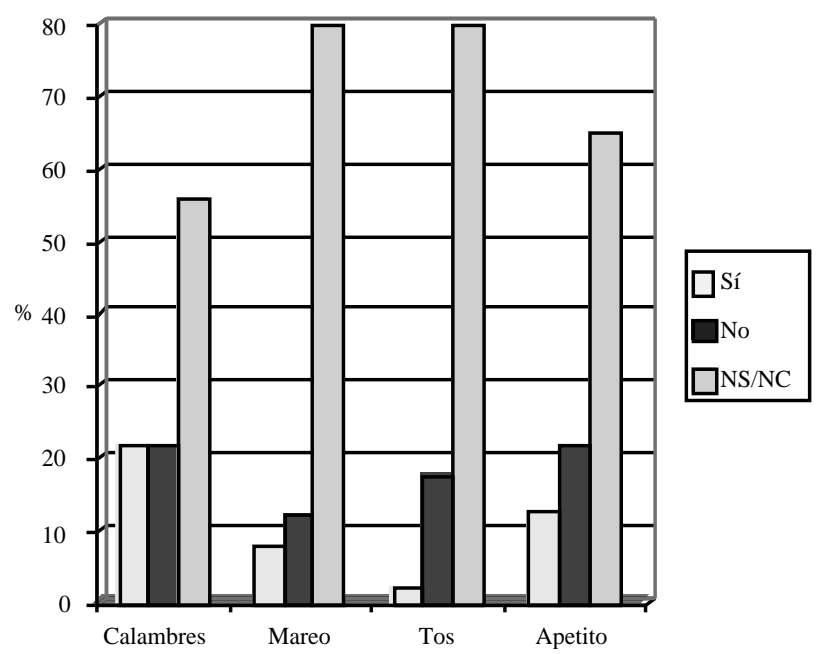

Fig. 2. Conocimiento de efectos secundarios farmacológicos (calambres en diuréticos; mareo y tos en IECAs); alteraciones del apetito en digoxina.

\section{DISCUSION}

Como se ha comentado al inicio del artículo, existen pocos estudios epidemiológicos en relación con la insuficiencia cardiaca, por ello se considera que los factores psicosociales rela- cionados con dicha enfermedad no han sido hasta el momento bien estudiados (7), hecho que se traduce en un desconocimiento de la importancia de los mismos en relación con el inicio, progreso y el manejo de esta enfermedad. Se escogieron mayores de 65 años por el mayor número de pacientes en ese rango de edad y la posible menor comprensión por parte de éstos sino existía una mínima explicación del facultativo encargado de su seguimiento. En primer lugar, se debe mencionar que el paciente sabe en que momento debe consultar al médico tras la aparición de una sintomatología determinada, así prácticamente la totalidad de los encuestados consultaría ante la presencia de disnea y/o edemas en los miembros inferiores. Es de destacar que la mayoría de los pacientes sabía perfectamente que el control de su peso era una medida necesaria para el manejo de su enfermedad. Así mismo, reconocían como beneficioso la realización de ejercicio en grado moderado y el uso restringido de sal. Estos datos corroboran que los pacientes poseen un adecuado nivel de información a través de su médico sobre el control de peso, realización de ejercicio moderado y hábito dietético con restricción de sal, lo que no siempre implica que lo lleven a cabo. Sin embargo, hay otros aspectos higiénico-dietéticos (8) sobre los cuales los pacientes no han sido informados adecuadamente como la ingesta hídrica moderada y el control de la diuresis de tal manera que un $50 \%$ de los pacientes encuestados no identificaron estas medidas como preventivas para el desarrollo de insuficiencia cardiaca, ya que el médico obvia, en ocasiones, comentar la paciente que controlen su diuresis. Además, es un consejo muy común por parte de los facultativos la necesidad de ingerir el mayor volumen de agua posible. Se han realizado estudios sobre la educación en medidas higiénico-dietéticas y de autocuidado (9-11) que abordan lo anteriormente expuesto aunque éstos no se han realizado en nuestro medio.

También se debe comentar, la existencia de estudios centrados en el manejo farmacológico de la insuficiencia cardiaca que demuestran la importancia vital de éste sobre el control de la misma. En el estudio realizado se recogen datos sobre los conocimientos del paciente acerca del tratamiento de la insuficiencia cardiaca. Una amplia mayoría de los encuestados reconoce la furosemida como diurético, quizás por ser éste uno de los fármacos más utilizados en el tratamiento de su enfermedad, así como por identificar, casi de forma inmediata, su efecto. Sin embargo, más de la mitad no reconocía los efectos secundarios relacionados con dicho medicamento, lo cual implica que quizás no se informa adecuadamente de la posibilidad de aparición de reacciones adversas. La digoxina también es identificada como un fármaco relacionado con el tratamiento de la insuficiencia cardiaca por los pacientes que la toman, aunque sus efectos secundarios también son desconocidos. Sin embargo, es mucho mayor el desconocimiento 
que existe sobre los IECAs tanto en su efecto como en la aparición de efectos secundarios, probablemente se puede relacionar este hecho con la reciente utilización de los mismos para el manejo de la insuficiencia cardiaca y de la hipertensión arterial asintomática a corto plazo. En cuanto a otros fármacos no relacionados con la insuficiencia cardiaca que son comúnmente utilizados, como pueden ser los AINEs, en general, se desconoce que no se puede utilizar cualquier tipo de éstos en presencia de la enfermedad. Las razones de este hecho radican en el difícil control por parte de los facultativos del abuso de los mismos por ser fármacos de fácil adquisición y de uso frecuente.

Además de los estudios señalados, existen otros dedicados a la medida del estado físico del paciente y de sus capacidades y habilidades para realizar las actividades cotidianas como determinantes en la progresión de su enfermedad. Otros, abordan el aspecto psicológico y la percepción de salud que el paciente tiene de sí mismo. Así, algunos de ellos determinan la importancia que el estado de ánimo depresivo puede tener en la evolución de la IC $(12,13)$. En la misma línea, algunos autores se centran en medir el apoyo social y familiar que reciben estos enfermos y como su propia socialización influye en la evolución de su patología de base (14). En última instancia se debe mencionar las diferencias que existen entre géneros en la evolución de la enfermedad, aspecto que no se aborda en la entrevista y, sobre el cual, si existen referencias bibliográficas, indicándose además que tras una intervención sobre los factores psicosociales $(15,16)$ y del manejo del tratamiento, la evolución de la IC se aproxima en ambos sexos (17).

Por el momento el proyecto presentado está muy limitado en el tiempo, pero se pretende prolongar el periodo de recogida de datos, para aumentar así la población estudiada y la diversidad de la misma: Un aspecto a reseñar del cuestionario utilizados, es la dificultad que en ocasiones tiene los pacientes para comprender las preguntas que se le realizaban, motivo por el cual se trataba de simplificar al máximo el contenido de las mismas, estando en la mayoría de los casos presente un familiar del paciente, asegurando así la fiabilidad de las fuentes.

Quizás el punto más interesante del estudio es el que permite que, en un futuro, se recojan de forma conjunta toda la información acerca de los factores psicosociales, que obligan a los pacientes a reingresar por IC. Tal vez se podría completar este estudio con un programa adecuado de la educación sanitaria adaptado a las necesidades de la población de riesgo a nivel de atención primaria (18). El abordaje de estos factores mejorará el pronostico de los pacientes con IC, reduciendo parámetros como su morbi-mortalidad y la incidencia de reingresos hospitalarios, así como facilitará de este modo el manejo de estos pacientes por facultativos tanto de primaria (19) como de especializada (20).

\section{Bibliografía}

1. Alonso Briales, JH, Gómez Robles JJ, Montiel Trujillo M. Insuficiencia cardiaca. Concepto, epidemilogía e historia natural. Medicine 1996; 16: 627-32.

2. Cowie MR, Mosterd A, Wood DA, Deckers JW, Poole-Wilson PA, Sutton GC. The epidemilogy or heart failure. Eur Heart J 1997; 18: 208-25.

3. McKee PA, Castelli WP, McNamara PM, Kannel WB. The natural history of congestive heart failure: The framingham study. N Engl J Med 1971; 285: 1441-6.

4. C. Sáenz de la Calzada. Protocolos de insuficiencia cardiaca. Ed Idepsa. Madrid 1992. pág. 5.

5. Conthe P, Lobos JM, Alonso A, Ruiz J. Insuficiencia cardiaca: lo que debo saber. Orientaciones dirigidas al paciente y a sus familiares. SEMI-semFyC. Ed Jarpyo. Madrid. 2001.

6. Hughes SL, Weaver FM, Giobbie-Hurder A. Effectiveness of teammanaged home-based primary care: a randomized multicenter trial. JAMA 2000; 284: 2877-85.

7. Kannel WB, Ho K, Tom T. Changing epidemiology features of cardiac failure. Br Heart J 1994, 72: 53-9.

8. Doba N, Tomiyama H, Nakayama T. Drugs, heart failure and quality of life: What are we achieving? What should be trying to achieve? Drugs Aging 1999; 14: 153-63.

9. Jaarsma T, Halfens R, Tan F. Self care and quality of life in patients with advanced heart failure: The effect of a supportive educational intervention. Heart Lung 2000; 29: 319-30.

10. Rockwell JM, Riegel B. Predictors of self care in persons with heart failure. Heart Lung 2001; 30: 18-25.

11. Jaarsma T, Halfens R, Huijer Abu-Saad H. Effects of education and support on self-care and resource utilization in patients with heart failure. Eur Heart J 1999; 20: 673-82.

12. Clarke SP, Frasure-Smith N, Lesperance F. Psycosocial factors as predictors of functional status at 1 year in patients with left ventricular dysfunction. Res Nurs Health 2000; 23: 290-300.

13. Freedland KE, Carney RM. Psychosocial considerations in elderly patients with heart failure. Clin Geriatr Med 2000; 16: 649-61.

14. Struthers AD, Anderson G, Donnan PT. Social deprivation increases cardiac hospitalizations in chronic heart failure independent of disease severity and diuretic non adherence. Heart 2000; 83:12-6.

15. Moser DK, Worster PL. Effect of psychosocial factors on physiologic outcomes in patients with heart failure. J Cardiovasc Nurs 2000; 14: 106-15.

16. Taylor DH Jr, Whellan DJ, Sloan FA. Effects of admission to a teaching hospital on the cost and quality of care of medicare beneficiaries. N Engl J Med 1999; 340: 309-10.

17. Riedinger MS, Dracup KA, Brecht ML. Quality of life in patients with heart failure: Do gender differencies exist?. Heart Lung 2001; 30: 105-16.

18. Lobos JM, Calvo S, Rojo G, Sánchez M, Muñoz Y, Jubete T. Evaluación de la calidad de la atención a pacientes con insuficiencia cardiaca crónica. Atención Primaria 1997; 20 (Supl. 1): 355.

19. Lobos JM, Díaz Sánchez S, Redondo R. Evaluación de la insuficiencia cardiaca desde la consulta de atención primaria. FCM 1997, 4: 415.

20. Grupo de trabajo de IC de la Sociedad Española de Medicina Interna. La insuficiencia cardiaca en los servicios de Medicina Interna (Estudio SEMI-IC). Med Clin (Barc) 2002; 118: 605-10. 